(RESEARCH ARTICLE)

\title{
Accurate heat detection and health monitoring of cattle by SCR sense neck collar
}

\author{
D. Indira ${ }^{1}{ }^{*}$, J. Suresh 2 and Y. Ravindra reddy ${ }^{3}$ \\ ${ }^{1}$ Assistant Professor, Dept of Livestock production management, College of veterinary science, Proddatur. \\ 2 Professor (Livestock Production Management) and Centre for Continuing Veterinary Education, Tirupati. \\ 3 Professor and Head, Dept of Livestock production management, College of veterinary science, Tirupati. \\ Sri Venkateswara Veterinary University, Tirupati, Andhra Pradesh, India
}

Publication history: Received on 15 November 2020; revised on 28 November 2020; accepted on 02 December 2020

Article DOI: https://doi.org/10.30574/wjarr.2020.8.3.0460

\begin{abstract}
Heat detection in dairy cattle is most important step in successful running of Dairy farms. But due to failure of heat detection at right time the dairy farms running under loss. To overcome this problem the Dodla Dairy at Pulivendula, Kadapa district introduced Heat Detection and Health monitoring SCR cSense Flex Neck Tag which aids in precision dairy farming by accurate detection of heat and monitoring animal activity status. After introduction of this new technology there were a lot of changes noticed and achieved the targeted results in terms of reproduction, health, nutrition, cow comfort and labor.
\end{abstract}

Keywords: Precision Dairy Farming; SCR cSense Neck Collar; Cow Comfort; Heat Detection

\section{Introduction}

Now a days almost all dairy farms facing the biggest problem that is detection of heat at right time in dairy cattle which is one of the deciding factors of running farm at profit or loss. Heat detection is a significant component of profitability in artificially bred dairy cattle but it has become the major limiting factor of reproductive performance in cattle (Lucy2007). Each missing of heat detection leads to economic loss, it may be negligible in initial stage of farm start up but accumulated effects the profitability of dairy farms and in turn it effects the dairy owners spirit to run the dairy farms.

High input and low output prices leading to lower productivity (Uddin et al 2010). To overcome this problem and to get profits in dairy farms there is a need for an alternative technique which helps in precision dairy farming. For this Dodla Dairy at Pulivendula, Kadapa District introduced the SCR cSense Flex Neck Tag which is the best solution to overcome the above said problems. It combines rumination and animal activity for heat detection and a revolutionary tool to monitor the dairy cows in real time (24 X 7).

The cSense Flex Tag collects information and transmits it to the Allflex - SCR system a few times per hour via RF technology, so the information in the system is up to date at all times. There are five major areas which will be monitored by this Tag. They are reproduction, health, nutrition, cow comfort and labor. These are the areas which brings profit to the dairy farms. Dodla Dairy at Pulivendula, Kadapa District after introduction of this technology had seen tremendous changes in their profits when compared to their previous profits. It's now a successful dairy farm in the above said aspects like reproduction, health, nutrition, cow comfort and labor efficiency.

*Corresponding author: D. Indira

Assistant Professor, Dept of LPM, C.V.Sc, Proddatur (A.P).

Copyright (C) 2020 Author(s) retain the copyright of this article. This article is published under the terms of the Creative Commons Attribution Liscense 4.0. 


\section{Decision making support for successful running of dairy farms by SCR cSense Flex Neck Tag}

\subsection{Detailed monitoring of Status of every cow}

The SCR cSense Flex Tag is neck Tag. It combines rumination and animal Activity for heat detection functionality to give dairy farms a revolutionary tool to monitor their cows in real time, 24 hours a day. Csens Flex Tag collects information and transmits it to the Allflex-SCR system a few times per hour via RF technology, so the information in the system is up-to-date at all times on all animals at dairy farm. Allflex Livestock Intelligence group monitoring applications provided dairy farm with timely and actionable insight that enables optimized decision-making for herd-wide health, wellbeing and productivity. Varied reports that reveal trends within groups enable dairy farm to make timely decisions relating to wellbeing, nutrition, heat abatement, and other farm management strategies.

\subsection{Nutrition monitoring}

Feeding efficiency management - Monitors variations in between cows groups and compares rumination trends. Highlights irregularities due to group changes, such as: feed delivery deviations, trying new indgredients, opening a new silage bag or bunk, ration adjustments, poor quality feed, etc. and analyzes long-term rumination trends. Cow response to nutrition and ration changes can be monitored with visible rumination reactions, changes and trends.

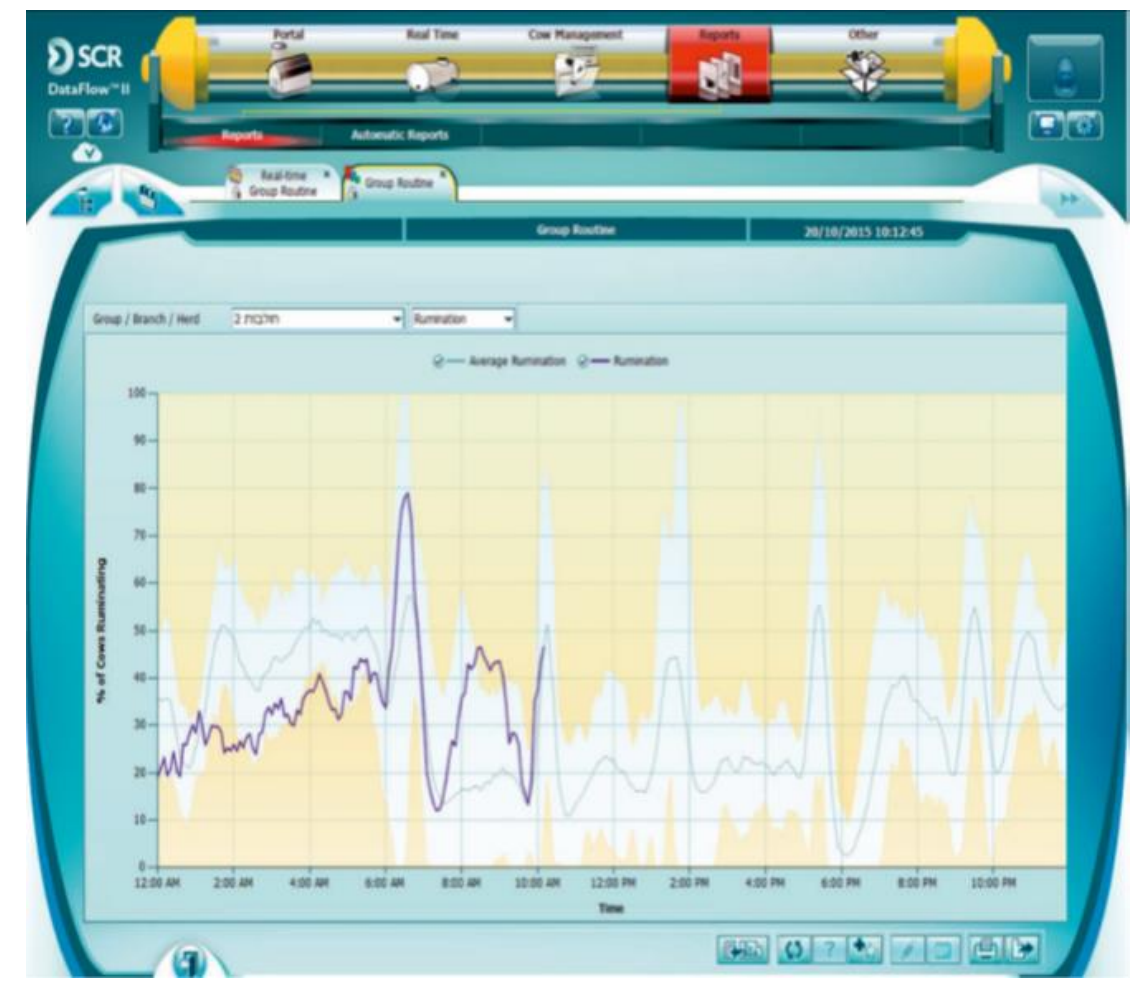

Figure 1 Group Routine Application - Rumination

\subsection{Health Monitoring}

Allflex Livestock Intelligence health monitoring applications empower dairy workers to detect health issues in individual cows early on and to quickly evaluate response to veterinary treatments. This enables informed and timely decision-making regarding intervention and treatment, helping avoid deterioration, reducing treatment costs, and reducing or preventing impact on milk production as well as mortalities. Improves health management with lifetime cow card history and individual health reports. Daily health reports list cows based on severity. Calving ease monitoring and reporting provides enhanced insight into calving diseases, reproduction problems, and herd key performance indicators (KPI). Distress monitoring across the whole lactation, Unusual activity or rumination at the group or herd level are highlighted by real-time updates, exposing issues group stress, spreading illness, group panic, and more. 


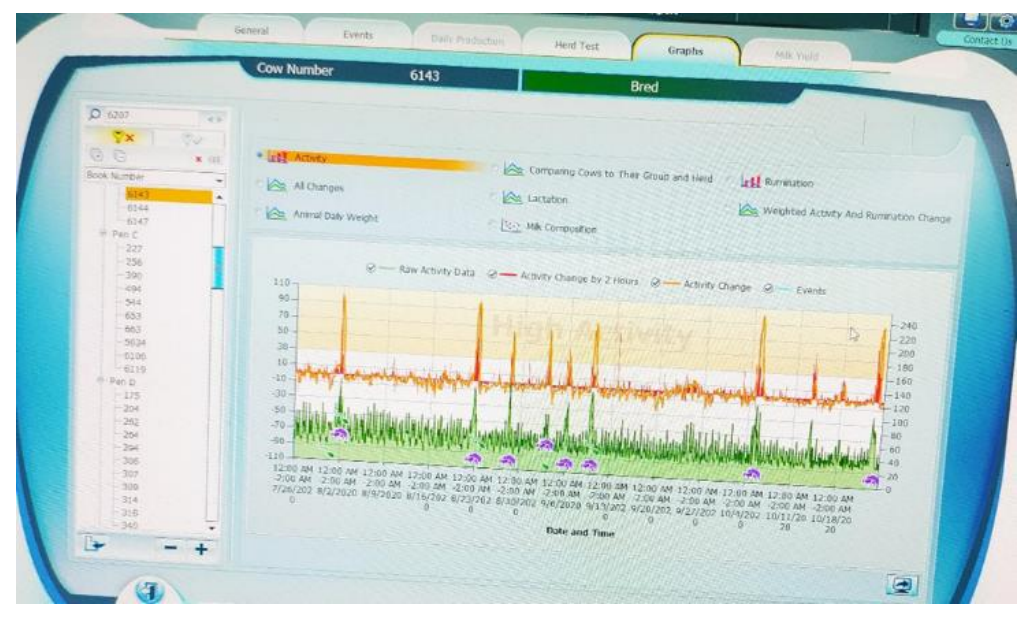

Figure 2 Animal activity graph

\subsection{Reproduction Monitoring}

Allflex Livestock Intelligence reproduction monitoring applications enable beef and dairy farmers to optimize conception rates, while reducing hormone use, labor and time. Leveraging behavior monitoring based on activity, rumination, eating and other proprietary key cow behaviors, our applications provide unmatched heat detection accuracy, delivering actionable insight in real time, with precise insemination timing guidance. Advanced Heat Index enhances heat detection and reduces false positives during pregnancy, decreasing checks for possible abortions. Separate reports for 'Cows to Inseminate' and 'Cows in Heat' based on voluntary waiting period. Facilitates management of your to-do list .Group Masked Heat report - Improves heat detection in groups with high activity or with frequent changes, such as grazing herd. Reports on cystic and anestrus cows - For enhanced monitoring ofreproduction problems .Calculates the probability of pregnancy and possible miscarriage for allpost-AI cows, allowing for earlier vet-checks to diagnose problems.Important key step in reproduction is early detection of heat and insemination at right time. Failure of detection of heat and late detection heat leads to missed conception followed by prolonged calving interval which leads to economic loss to dairy farms (Rao TKS et al). The more number of animals involved the more loss to the farms. Usually dairy workers should monitor the cattle regularly at early in the morning and evening. But at dairy farms especially large dairy farms monitoring of detection of heat difficult due to silent heat, expressed heat signs may not be identified in large cattle population. If identified also that can't be a successful conception because of the early or late insemination leads to missed conception.

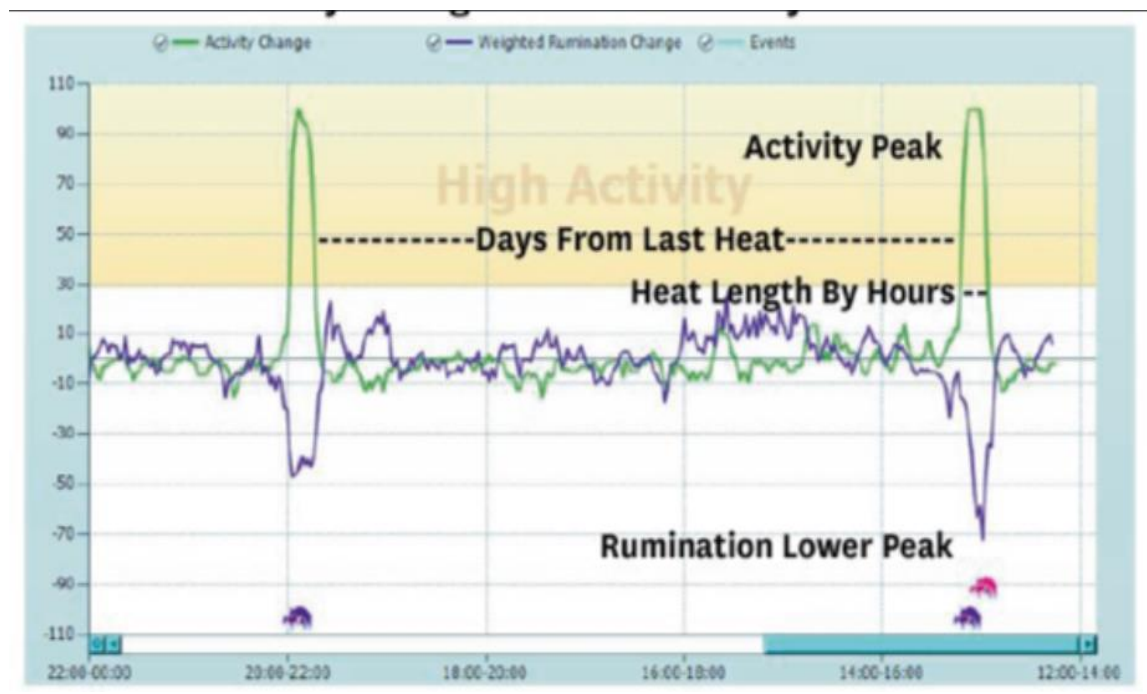

Figure 3 Pregnancy probability graph

\subsection{Calving}

Pre-Calving Distress Alert - Real-time alerts and essential insight on cows experiencing unusual stress around expected calving. Post-Calving Distress Alert - Minimize the impact of a calving disease with early warnings of a post-calving 
problem. The SCR system closely monitors each cow during the initial days after calving and provides a specific distress alert in the event that rumination deviates beyond what is normal.

\subsection{Temperature and Humidity Index (THI)}

Detect heat stress and minimize effects on your cows by monitoring temperature and humidity.Climate and humidity sensors connect and report the temperature and humidity into the SCR Heatime® Pro System. Notification when the THI exceeds or drops below the target In addition to the direct benefit to animal welfare, the application allows for early intervention for cooling and ration changes

\subsection{Heat stress and group monitoring module}

Now with group panting, eating and rumination, combined on the same reports and graphs. Compare panting with rumination, eating and THI to identify the impact of heat stress on cow comfort and production. Monitors THI to aide formulation of improved daily and long-term heat abatement strategies Group reports help identify the seasonal impacts of heat stress. Group heat stress graph provides a within-the-day heat stress trend and its impact on rumination and eating.

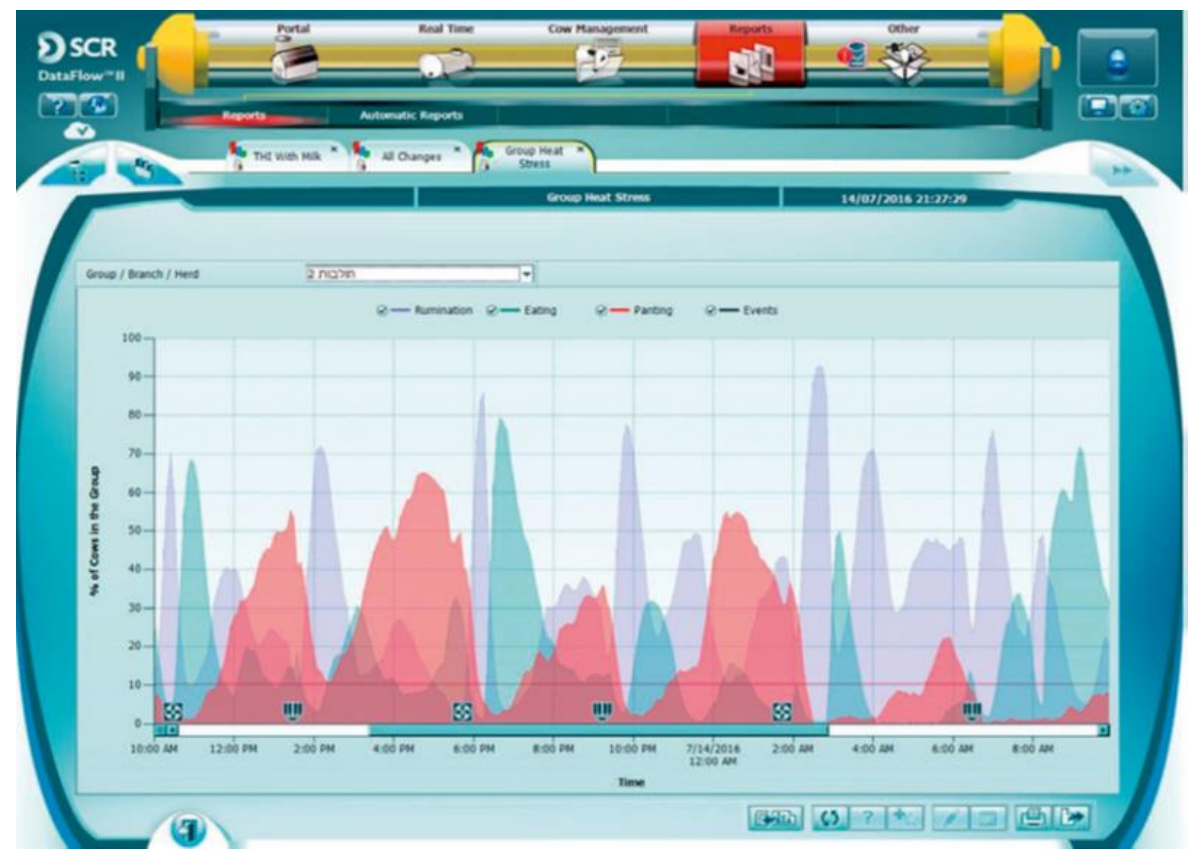

Figure 4 Group heat stress graph

\section{Conclusion}

SCR cow monitoring systems ensures decision making support for improved herd management. It manages the herd by measuring activity, rumination and behavioral pattern of each cow because of this we could monitor the status of every cow at any given time, information can be evaluated on both an individual cow and a group basis. This allows for early identification of health, reproduction, nutrition and wellbeing issues.

\section{Compliance with ethical standards}

\section{Acknowledgments}

All authors would like to thank Dodla dairy, Pulivendula for their support in finishing this manuscript

\section{Disclosure of conflict of interest}

All authors declaring that there is no conflict of interest to declare 


\section{References}

[1] Rao TKS, Kumar N, Kumar P, Chaurasia S, Patel NB. Heat detection techniques in cattle and buffalo. 2013; 6(6):363-369.

[2] Lucy MC. Fertility in high-producingdairy cows: reasons for decline and corrective strategies for sustainable improvement.SocReprodFertil Suppl. 2007; 64: 237-254.

[3] Uddin MM, Sultana MN, Ndambi OA, HemmeT, Peters KJ. A farm economic analysis in different dairy production systems in Bangladesh. Livestock Research for Rural Development. 2010; 22: 122. 